\title{
25 Research Soure \\ Development of a Web Tool to Calculate the Cumulative Dose of Glucocorticoids
}

\section{Nuria Montero-Pastor}

Sociedad Española de Reumatología

Jesús Sánchez-Costa

Sociedad Española de Reumatología

Mercedes Guerra-Rodríguez

Sociedad Española de Reumatología

Clara Moriano

Complejo Asistencial Universitario de León

Javier Loricera

Marqués de Valdecilla University Hospital

Federico Díaz-González ( $\square$ federico.diaz.gonzalez@gmail.com )

Departamento de Medicina Interna, Dermatología y Psiquiatría. Universidad de La Laguna. Spain

\section{Research Article}

Keywords: Glucocorticoids, cumulative dose, minimizing bias, treatment period

Posted Date: January 18th, 2021

DOI: https://doi.org/10.21203/rs.3.rs-146726/v1

License: (c) (i) This work is licensed under a Creative Commons Attribution 4.0 International License.

Read Full License 


\section{Abstract}

Glucocorticoids are associated with many serious side effects, most of which are related to dosing and time of use. Correct assessment of cumulative dose, especially in patients undergoing long-term treatment, is essential to determine the risk of undesirable glucocorticoid-related events. Unfortunately, there is no standard method currently available for determining exposure to glucocorticoids in daily clinical practice. The aim of this work was to create an easy-to-use free-access web application to help clinicians and researchers calculate, in a systematic way, the cumulative dose of corticosteroids in their patients. The total cumulative dose is calculated as the sum of all periods of treatment with different doses of oral or intravenous corticosteroids, and is expressed as the equivalent dose of prednisone in mg. The doses of glucocorticoids during the periods without information are estimated by first determining the average doses between the previous and subsequent periods for which such information is available, and then multiplying that average dose by the number of days of the period. If the day on which glucocorticoids were discontinued is unknown, it is assumed to be the day before the start of the next known treatment period. For unknown start dates, the day after the end of the known prior period is used. In conclusion, this tool simplifies and, through the systematic application of assumptions when information is incomplete, standardizes the glucocorticoids cumulative dose calculation, minimizing bias in the assessment of glucocorticoid-related side effects in the context of daily clinical practice.

\section{Introduction}

Glucocorticoids are a foundation of effective therapy for many inflammatory and autoimmune human disorders ${ }^{1-5}$. Despite their undoubted beneficial therapeutic properties, the use of these compounds is associated with many well-known harmful adverse events (revised in ${ }^{6}$ ), which constitutes the main limitation preventing their widespread use. Most glucocorticoids adverse events are related to dosing and treatment duration, two variables that determine the cumulative dose and consequently the exposure of patients to these compounds ${ }^{7}$. It has been demonstrated that glucocorticoid-related adverse events can be mitigated by certain actions, such as using less potent agents or by administering them at the lowest dose and over the shortest time frame possible ${ }^{8,9}$. In this way, correct assessments of cumulative doses of glucocorticoids is particularly important to determine the risk of suffering undesirable effects.

Due to long-term use and frequent dose variations in response to changes in disease activity, information about glucocorticoids is often inaccurately and incompletely recorded in patient medical records. Glucocorticoid exposure assessments are often carried out by physicians in their daily clinical practice using non-systematized estimations. This makes it particularly difficult to conduct inter-study comparisons of the side effects caused by glucocorticoids, or even to analyze the influence of glucocorticoid treatments on the side effects caused by other concomitant medications. Although several tools have been designed to assess the cumulative dose of glucocorticoids ${ }^{10,11}$, no consensus has emerged on how best to standardize the assessment of glucocorticoid exposure when using data from daily clinical practice. 
The aim of this work is to provide an easy-to-use method to standardize the assessment of the cumulative dose of glucocorticoids using information obtained from patients' daily clinical practice records. We believe that the use of this tool will reduce bias in the assessment of glucocorticoid exposure between different studies.

\section{Methods}

This section presents the general reasoning, including a description of the assumptions in case of incomplete information, on which this proposal to standardize the assessment of glucocorticoids' exposure is based. To facilitate the use of this tool, a free software application based on this proposal in English and Spanish, designated CORTISER+, has been developed (https://cortiser.ser.es) (see supplementary text).

In this proposal, oral and intravenous (iv) doses of glucocorticoids were calculated separately. The total cumulative dose of glucocorticoids, expressed as the total prednisone-equivalent dose in mg (Table 1), was calculated by adding the cumulative partial doses of the different iv-administered boluses to the cumulative partial doses produced by each one of the different periods during which the patient was taking glucocorticoids orally.

Table 1. Glucocorticoid equivalencies.

\begin{tabular}{|ll|}
\hline Drug & Conversion factor \\
\hline Deflazacort & 0.83 \\
\hline Methylprednisolone & 1.25 \\
\hline Prednisone/prednisolone & 1 \\
\hline
\end{tabular}

To estimate the dose of intravenous steroids, information about the strength of each bolus, the number of boluses, and the start and end dates of treatment had to be entered into the computer application. The application then simply multiplied the strength by the number of boluses and transformed the result into mg of prednisone.

Oral glucocorticoids were calculated using information regarding the strength of the daily dose, the type of compound and the start and end dates of treatment. The total days of treatment were calculated as the difference between the end date and the start date of treatment. In the optimal situation in which all information is available, the application simply multiplied the dose administered daily by the number of days of treatment and transformed the result into mg of prednisone, as detailed in Table 1. However, information regarding dosage, days of treatment, or both, of oral glucocorticoids is often incomplete or unavailable in medical records. The proposed method makes assumptions to estimate the cumulative dose of glucocorticoids, when: 
1. The strength of the daily dose and treatment start date are known, but not the end date. In this situation, the end date will be estimated as the day before the start date of the next known treatment period (Fig. 1A). To calculate the cumulative dose, the number of days between the known start date and the estimated end date is calculated and then multiplied by the daily dose. The known data must be included in the application and the unknown data is left blank.

2. The strength of the daily dose and the end date of the treatment period are known, but not the start date. The start date is estimated as the day after the known end date of the previous period (Fig. 1B). To calculate the cumulative dose for this period, the number of days between the known end date and the estimated start date is calculated and multiplied by the daily dose. Only known data should be entered into the calculator.

3. The start and end doses of glucocorticoids are different and the variations in dosing during that period are unknown (Fig. 1C). To calculate the cumulative dose for this period, the average between the initial and end doses of glucocorticoids is multiplied by the duration of the period.

To correctly use the calculator, one should adhere to the following recommendations:

1. The date of glucocorticoids treatment start is essential. If investigators do not know this date, the date from which the cumulative dose is assessed should be utilized.

2. The end date of glucocorticoids treatment is also mandatory. If the investigator does not know this date, the last known date glucocorticoids were used or the date the cumulative dose assessment ended should be utilized.

3. When the patient takes glucocorticoids on alternate days or different doses on consecutive days, it will be assumed that the strength of the daily dose for that period will be equal to the average of two consecutive days.

4. Periods when the patient is not taking glucocorticoids should be included as daily dose $=0$. This allows for a distinction between periods when the patient was not on glucocorticoid treatment and periods when it is unknown whether the patient was using them.

\section{Discussion}

The main aim of this proposal is to provide an easy-to-use tool to standardize the assessment of the cumulative dose of glucocorticoids. This calculator can help minimize the bias that non-standardized assessments of long-term glucocorticoid use cause in the evaluation of the side effects of these compounds, both in daily clinical practice and in clinical research.

The chronicity of glucocorticoid use and the variations in glucocorticoid dosing over time due to changes in the inflammatory process explain why information on these compounds is often incompletely or inaccurately collected in medical records. While there are many rheumatology conditions in which the cumulative dose of steroids have been examined as a confounder of comorbidities and response to treatment ${ }^{10-13}$, and though some researchers have proposed their own tools ${ }^{14-20}$, there is no 
standardized method for calculating the cumulative dose of glucocorticoids. This lack of standardization means that data on cumulative doses of glucocorticoids are not comparable between studies.

The main limitation of this tool is that when no specific information is available in the clinical histories (mainly concerning oral administration), it becomes necessary to make assumptions about doses and exposure times to glucocorticoids based on available data. However, since these assumptions follow a systematic procedure, it is possible to compare cumulative doses of glucocorticoids individually among patients and globally among studies when using this tool.

We believe that the widespread use of this calculator may help to minimize the bias that results from nonstandardized cumulative dose assessments of glucocorticoids both in clinical practice and clinical studies.

\section{Declarations}

\section{Acknowledgments}

This application is supported by Roche.

\section{Authors' contributions}

N.M-P, J.T-S and F.D-G designed the method and application, tested the application and wrote the final version of the manuscript. M.G-R. did the literature search. C.M and J.L tested the application and wrote the preliminary version of the manuscript. All the authors have discussed, corrected and approved the final version of the manuscript.

\section{Competitive Financial Interests}

The authors state that there are no competing financial interests.

\section{Bibliography}

1 Conti, F., Morelon, E. \& Calmus, Y. Immunosuppressive therapy in liver transplantation. J Hepato/39, 664678, doi:10.1016/s0168-8278(03)00428-8 (2003).

2 Coutinho, A. E. \& Chapman, K. E. The anti-inflammatory and immunosuppressive effects of glucocorticoids, recent developments and mechanistic insights. Mol Cell Endocrino/335, 2-13, doi:10.1016/j.mce.2010.04.005 (2011).

3 Kamen, D. L. \& Zollars, E. S. Corticosteroids in Lupus Nephritis and Central Nervous System Lupus. Rheum Dis Clin North Am42, 63-73, viii, doi:10.1016/j.rdc.2015.08.008 (2016).

4 Mathian, A. et al. Withdrawal of low-dose prednisone in SLE patients with a clinically quiescent disease for more than 1 year: a randomised clinical trial. Ann Rheum Dis79, 339-346, doi:10.1136/annrheumdis- 
5 Mullerova, H., Cockle, S. M., Gunsoy, N. B., Nelsen, L. M. \& Albers, F. C. Clinical characteristics and burden of illness among adolescent and adult patients with severe asthma by asthma control: the IDEAL study. J Asthma, 1-12, doi:10.1080/02770903.2019.1708095 (2020).

6 Oray, M., Abu Samra, K., Ebrahimiadib, N., Meese, H. \& Foster, C. S. Long-term side effects of glucocorticoids. Expert Opin Drug Saf15, 457-465, doi:10.1517/14740338.2016.1140743 (2016).

7 McDonough, A. K., Curtis, J. R. \& Saag, K. G. The epidemiology of glucocorticoid-associated adverse events. Curr Opin Rheumato/20, 131-137, doi:10.1097/BOR.0b013e3282f51031 (2008).

$8 \mathrm{Liu}, \mathrm{D}$. et al. A practical guide to the monitoring and management of the complications of systemic corticosteroid therapy. Allergy Asthma Clin Immuno/9, 30, doi:10.1186/1710-1492-9-30 (2013).

9 van der Goes, M. C., Jacobs, J. W. \& Bijlsma, J. W. The value of glucocorticoid co-therapy in different rheumatic diseases-positive and adverse effects. Arthritis Res Ther16 Suppl 2, S2, doi:10.1186/ar4686 (2014).

10 Best, J. H., Kong, A. M., Unizony, S., Tran, O. \& Michalska, M. Risk of Potential Glucocorticoid-Related Adverse Events in Patients with Giant Cell Arteritis: Results from a USA-Based Electronic Health Records Database. Rheumatol Ther6, 599-610, doi:10.1007/s40744-019-00180-9 (2019).

11 Gale, S. et al. Risk Associated with Cumulative Oral Glucocorticoid Use in Patients with Giant Cell Arteritis in Real-World Databases from the USA and UK. Rheumatol Ther5, 327-340, doi:10.1007/s40744018-0112-8 (2018).

12 del Rincon, I., Battafarano, D. F., Restrepo, J. F., Erikson, J. M. \& Escalante, A. Glucocorticoid dose thresholds associated with all-cause and cardiovascular mortality in rheumatoid arthritis. Arthritis Rheumato/66, 264-272, doi:10.1002/art.38210 (2014).

13 van Staa, T. P. et al. A simple score for estimating the long-term risk of fracture in patients using oral glucocorticoids. QJM98, 191-198, doi:10.1093/qjmed/hci029 (2005).

14 Amiche, M. A. et al. Impact of cumulative exposure to high-dose oral glucocorticoids on fracture risk in Denmark: a population-based case-control study. Arch Osteoporos13, 30, doi:10.1007/s11657-018-0424-x (2018).

15 Balasubramanian, A. et al. Glucocorticoid Exposure and Fracture Risk in a Cohort of US Patients With Selected Conditions. J Bone Miner Res33, 1881-1888, doi:10.1002/jbmr.3523 (2018).

16 Dixon, W. G. et al. Immediate and delayed impact of oral glucocorticoid therapy on risk of serious infection in older patients with rheumatoid arthritis: a nested case-control analysis. Ann Rheum Dis71, 1128-1133, doi:10.1136/annrheumdis-2011-200702 (2012). 
17 Florance, J., Hemke, R., Chang, C. Y., Torriani, M. \& Bredella, M. A. Effects of intra-articular corticosteroid injections on lumbar trabecular density. Skeletal Radio/49, 787-793, doi:10.1007/s00256019-03362-2 (2020).

18 Mebrahtu, T. F. et al. Dose dependency of iatrogenic glucocorticoid excess and adrenal insufficiency and mortality: a cohort study in England. J Clin Endocrinol Metab, doi:10.1210/jc.2019-00153 (2019).

19 Palmsten, K. et al. Oral corticosteroid use during pregnancy and risk of preterm birth. Rheumatology (Oxford)59, 1262-1271, doi:10.1093/rheumatology/kez405 (2020).

20 van Vollenhoven, R. F. et al. Cumulative Corticosteroid Dose Over Fifty-Two Weeks in Patients With Systemic Lupus Erythematosus: Pooled Analyses From the Phase III Belimumab Trials. Arthritis Rheumato/68, 2184-2192, doi:10.1002/art.39682 (2016).

\section{Figures}


A

Start date of

End date of

treatment A

treatment A

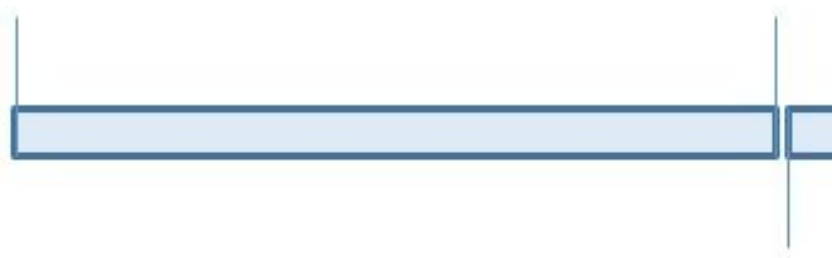

Start date of

End date of

treatment B

treatment B

B



$C_{\text {treatment A }}^{\text {Start date of }}$

End date of

treatment A



Unknown dose

Start date of

End date of

treatment B

treatment B

\section{Figure 1}

Schematic representation of different cases of treatments where the dose, end date and start date of treatment can be known, or not. Green color indicates known data and red color unknown data.

\section{Supplementary Files}

This is a list of supplementary files associated with this preprint. Click to download. 
- Supplementarymaterial.docx

Page $9 / 9$ 